\title{
A Local Electricity Trading Market: Security Analysis
}

DOI:

10.1109/ISGTEurope.2016.7856269

\section{Document Version}

Accepted author manuscript

Link to publication record in Manchester Research Explorer

\section{Citation for published version (APA):}

Mustafa, M. A., Cleemput, S., \& Abidin, A. (2016). A Local Electricity Trading Market: Security Analysis. In 2016 IEEE PES Innovative Smart Grid Technologies Conference Europe (ISGT-Europe) IEEE.

https://doi.org/10.1109/ISGTEurope.2016.7856269

\section{Published in:}

2016 IEEE PES Innovative Smart Grid Technologies Conference Europe (ISGT-Europe)

\section{Citing this paper}

Please note that where the full-text provided on Manchester Research Explorer is the Author Accepted Manuscript or Proof version this may differ from the final Published version. If citing, it is advised that you check and use the publisher's definitive version.

\section{General rights}

Copyright and moral rights for the publications made accessible in the Research Explorer are retained by the authors and/or other copyright owners and it is a condition of accessing publications that users recognise and abide by the legal requirements associated with these rights.

\section{Takedown policy}

If you believe that this document breaches copyright please refer to the University of Manchester's Takedown Procedures [http://man.ac.uk/04Y6Bo] or contact uml.scholarlycommunications@manchester.ac.uk providing relevant details, so we can investigate your claim.

\section{OPEN ACCESS}




\title{
A Local Electricity Trading Market: Security Analysis
}

\author{
Mustafa A. Mustafa, Sara Cleemput, and Aysajan Abidin \\ KU Leuven, ESAT-COSIC and iMinds, \\ Kasteelpark Arenberg 10, B-3001 Leuven-Heverlee, Belgium \\ Email: \{firstname.lastname\}@esat.kuleuven.be
}

\begin{abstract}
This paper proposes a local electricity trading market and provides a comprehensive security analysis of this market. It first presents a market for electricity trading among individual users, and describes the different entities and the interactions among them. Based on this market model and the interactions, the paper analyses security problems and potential privacy threats imposed on users, which leads to the specification of a set of security and privacy requirements. These requirements can be used to guide the future design of secure local electricity trading markets or to perform a risk assessment of such markets.

Index Terms-Smart Grid, Electricity Trading, Threat Analysis, Privacy, Security, Requirements, Risk Assessment.
\end{abstract}

\section{INTRODUCTION}

The Smart Grid (SG) is the next generation electrical grid that can support bi-directional electricity and communication flows [1]. The aim of the SG is to increase the grid's efficiency and reliability as well as to seamlessly integrate a vast number of Renewable Energy Sources (RESs). These RESs are spread across the grid and have intermittent electricity outputs that are hard to predict. The electricity they generate is usually consumed by their owners (i.e., private users).

However, in case RESs produce more electricity than their respective users need, the excess electricity is automatically injected back into the grid. Unfortunately, in current electricity markets, users receive no (or limited) remuneration for the electricity exported. For example, in Flanders (Belgium) users receive no payments for the electricity they export to the grid [2], whereas in the UK users automatically sell their exported electricity to their supplier for a fixed price which is much lower than the retail price [3]. Thus, a potential local electricity market that allows users to trade electricity among themselves could increase the users' financial well-being.

In addition, electricity trading on a local level could also be beneficial to the grid itself [4]. For example, the electricity exchange between users nearby can significantly reduce the amount of electricity that is wasted during the transmission over the distribution lines. Moreover, performing a local electricity trade contributes further to the autonomy of microgrids while reducing the demand and reliance on the main grid. Therefore, it is of interest to devise a market that enables such local electricity trading between users who have excess electricity (i.e., sellers) and users and/or suppliers who are in a need of electricity (i.e., buyers).

Several papers [5]-[7] have proposed market models which allow users to negotiate a price and sell their excess electricity to different suppliers. These models are good for RES owners as the users can potentially increase their revenues by selling electricity at a higher price. However, users without any RES would not benefit from these market models as they still be buying electricity from their contracted suppliers.

In this paper we propose a local electricity market which allows users to trade their excess electricity with other users and/or suppliers. We also analyse the security and privacy implications of such local markets. The main contributions of this paper are two-fold.

- Firstly, it proposes a novel local electricity market which allows (i) RES owners to sell their excess electricity to other users or suppliers and (ii) non-RES users to bid and buy electricity directly from RES users at a trading price determined by the market.

- Secondly, it performs a threat analysis of the proposed electricity market in order to specify a set of security and privacy requirements. These requirements will need to be satisfied in order to allow users to trade electricity among themselves in a secure and privacy-preserving manner.

The remainder of this paper is organised as follows: Section II provides some background information and discusses related work. Section III proposes a local electricity trading market. Section IV analyses potential security threats/attacks in the proposed market. Section V specifies a set of security and privacy requirements, and Section VI concludes the paper.

\section{BACKGROUND AND RELATED WORK}

\section{A. Existing Electricity Markets}

As shown in Fig. 1, electricity is typically traded in three markets: a wholesale, a balancing and a retail market [8].

The wholesale market is used for trading electricity in bulk between suppliers (i.e., utility companies) and generators of electricity (GENs). It is a competitive market, i.e., suppliers buy electricity at a price they are willing to pay and generators sell electricity for a price they are willing to accept. The final price is reached by negotiations. On the wholesale market electricity is traded for short, e.g., half-hourly, time periods referred to as settlement periods. Moreover, electricity is not traded in real-time. All contracts for each settlement period are frozen at some point in advance, called the gate closure. After the gate closure electricity trading on the wholesale market for the corresponding settlement period is not permitted. 


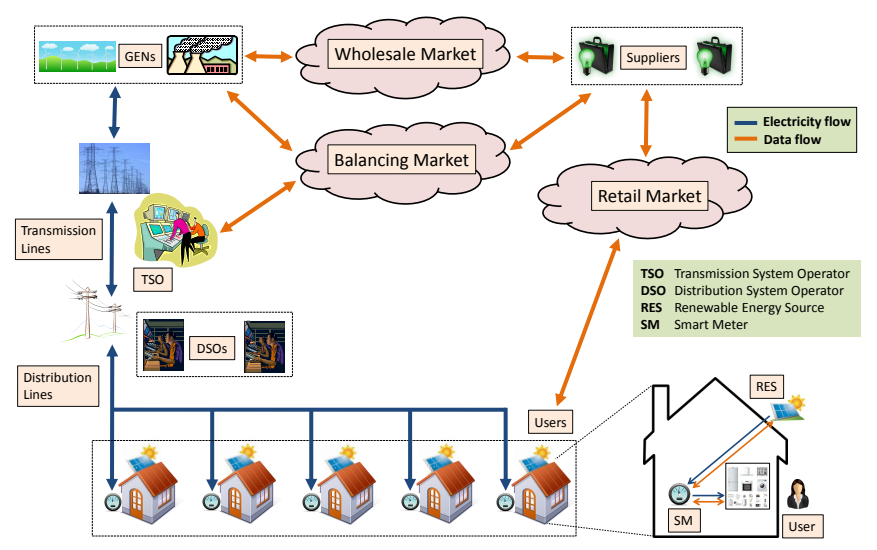

Fig. 1. Electricity tradings in liberalised electricity markets.

The balancing market is used for trading electricity in realtime and is controlled by a transmission system operator (TSO). The TSO uses the market to match the supply of electricity with the demand and/or to alleviate any issues on the transmission network during a settlement period. To overcome these types of imbalance, adjustments of the supply/demand should be performed. To perform these adjustments, the TSO has a range of different balancing services, such as buying extra electricity on the balancing market, or activating strategic reserves. Usually, the TSO selects the most efficient, economic and reliable balancing service available.

The retail market is used for trading electricity between users and suppliers. It is a competitive and dynamic market, i.e., individual users can choose their supplier and switch suppliers as often as they wish. However, unlike the wholesale market where the electricity price can vary in each settlement period, in the retail market users have fixed tariffs, usually one for the electricity consumed at peak times (during the day) and another cheaper one for the electricity consumed at off-peak times (at night). The market arrangements for the electricity injected back into the grid by RES users are different for each country. As mentioned earlier, in Flanders users receive no payments for the electricity they export [2], whereas in the UK users automatically sell their exported electricity to their contracted supplier for a fixed price lower than the retail price. For example, the export tariff in the UK is 4.77 pence per kilowatt hour (p/kWh) [3], whereas the average import (i.e., retail) price users pay is $13.9 \mathrm{p} / \mathrm{kWh}$ [9].

It is clear that the monetary rewards which RES users receive for the electricity they export are low (or non-existing). To improve this situation new market models are needed.

\section{B. Electricity Markets Proposed in the Literature}

Yaagoubi and Mouftah [10] proposed a market that provides buyers with the ability to find a seller with cheaper electricity prices and enough supply in order to minimize their electricity bill while taking into account transmission costs. The market is based on a game where a modified regret matching procedure is played by buyers to determine the best seller. Vytelingum et al. [5] proposed a market for trading electricity between users and microgrids that is based on continuous double auction and that automatically manages the congestion within the system by pricing the flow of electricity. Lee et al. [6] proposed direct trading between small-scale electricity suppliers and users without going through the traditional retailers. The trading price is determined instantaneously based on the number of participants and statistical information about electricity supply/demand. The authors showed that such direct trading of electricity could be beneficial for both sellers and buyers, as the price of the electricity traded directly lies between the wholesale and retail price. Lee et al. [7] proposed a distributed model for electricity trading among microgrids in a competitive market. Under their approach, sellers lead the competition by independently deciding the amount of electricity for sale, whereas buyers follow the sellers' actions by independently submitting a unit price bid to the sellers. Their game-theoretic study provides an incentive for electricity trading among microgrids.

Tushar et al. [11] argued for the benefits of allowing RES users to choose the price at which they are willing to sell their excess electricity. Moreover, to have a sustainable electricity market and encourage users to buy electricity from RESs, the authors suggest that the price of the electricity produced by RES users should be lower than the price of the electricity available from the grid, i.e., the contracted supplier of users. In addition, the authors argued that players with a larger amount of surplus electricity might be more interested in selling all the electricity for higher return, and thus will be flexible in reducing their asking price. On the other hand, a user with a small electricity surplus may not be keen to sell electricity unless the price per unit is sufficiently high as otherwise the expected return will be small. Also, it is rational to assume that each user wants to increase the price per unit of electricity they sell for as much as possible. Ampatzis et al. [12] proposed a local electricity market for coordinating RESs. The authors investigated discriminatory and uniform pricing. The former means that each trade has a different trading price which is a function of the supply/demand price of the matched bids; the latter means that there is one trading price for all trades derived from the aggregation of the supply/demand bids. The authors concluded that the uniform pricing increases revenues for users. Bayram et al. [13] provided an overview of distributed energy trading concepts in smart grid. They also argued that the biggest motivation for users to participate in such markets is the cost savings and increased profits for users.

Unlike the aforementioned market models, we propose a local electricity market which allows not only local users but also suppliers to trade excess electricity generated by RESs. Moreover, we specify a set of functional requirements and provide potential interactions among different entities in our model, such that the model is suited for the existing liberalised electricity markets. In addition, we perform a comprehensive risk and threat analysis to identify the potential risks, and specify a set of security and privacy requirements for such a market model in order to mitigate the identified threats. 


\section{A Local Electricity Trading Market}

This section details the system model, functional requirements, possible interactions among entities and the benefits of our proposed local market for trading electricity.

\section{A. System Model}

As shown in Fig. 2, our proposed local electricity trading market consists of the following entities.

- Renewable Energy Sources (RESs) are mini generators (e.g., solar panel) located on users' premises. The electricity they generate is usually consumed by their owners. However, surplus electricity may be injected into the grid.

- Smart Meters (SMs) are advanced metering devices which can measure the amount of electricity flowing in both directions (from the grid to the house and vice versa) and perform two-way communications with other entities.

- Users are people who consume electricity and pay for it. In addition, they are rational, i.e., they try to reduce their electricity bills by looking for the cheapest possible electricity source; if they own RESs, they try to sell the excess electricity at the highest possible price.

- Suppliers are responsible for supplying electricity to all users who could not get enough electricity from their own RES or on the local market. They buy this electricity from generators and sell it to users. They are also obliged to buy the electricity their customers inject into the grid, if the customers have not traded it on the local market.

- Distribution System Operator (DSO) is responsible for maintaining and managing the distribution network in a particular region. It also charges the suppliers distribution network fees based on the electricity consumption/provision data of the suppliers' customers in this region.

- Transmission System Operator (TSO) is responsible for maintaining the transmission network, balancing the grid and charging suppliers transmission network fees based on the electricity consumption/provision data of the suppliers' customers in the grid.

\section{B. Functional Requirements}

To be adopted by users and be suitable to the existing liberalised electricity markets, our proposed local electricity trading market should satisfy the following functional requirements.

- Each user should pay (be paid) for the electricity she buys (sells) in the local electricity market via her supplier.

- Each supplier should

- charge its customers only for the electricity supplied to them from the grid, i.e., by the supplier;

- pay to its customers only for their exported electricity that was not traded in the local electricity market, i.e., automatically sold to the supplier;

- cooperate with other suppliers to assist users in settling payments among users for electricity traded in the local market; and

- access the imported and exported electricity from the grid by all its customers located in a DSO region

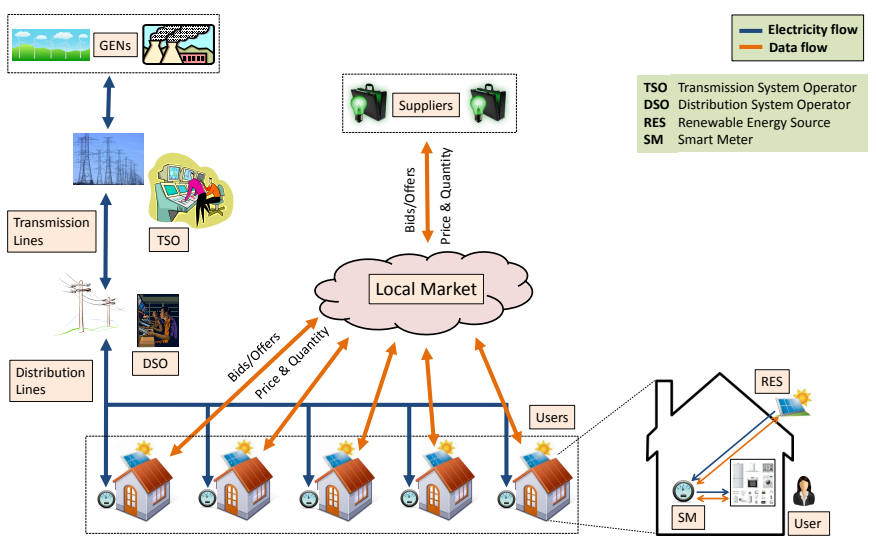

Fig. 2. A proposed local market for trading electricity from RESs.

for each settlement (electricity trading) period, such that it can predict its customers demand accurately. This is important in order to avoid imbalance fines and to be assured that it pays the correct distribution network fee to the corresponding DSO.

- For each settlement period, the DSO should access

- the imported and exported electricity by all users in its region of operation, so it can better manage the distribution network in the region; and

- the imported and exported electricity by all users per supplier in the region, so it can split distribution network fees fairly among suppliers.

- For each settlement period, the TSO should access the imported and exported electricity by all users in a DSO region so it can better balance the grid and split transmission network and balancing fees fairly among suppliers.

\section{Interactions among Entities}

Potential message types and interactions among the entities in a local electricity market are described next.

1) Submitting offers/bids: Prior to a trading period, users and suppliers submit their offers/bids to the local electricity market. With these offers/bids users inform the market how much electricity and for what price per unit they can sell or buy electricity during the trading period. Users and suppliers are free to set their own offer/bid prices per electricity unit. However, to be appealing to potential buyers/sellers, these prices should be between the export and retail price offered by the suppliers.

2) Setting a trading price: As shown in Fig. 3, the local market performs a double auction trading as follows.

- It sorts the sellers (RES owners) in an increasing order of their offer prices and the buyers (users and suppliers) in a decreasing order of their bid prices. Whenever two or more buyers/sellers have equal offer/bid prices, the local market groups them into a single virtual buyer/seller.

- It generates the supply and demand curve. The intersection of these two curves is used to (i) determine 


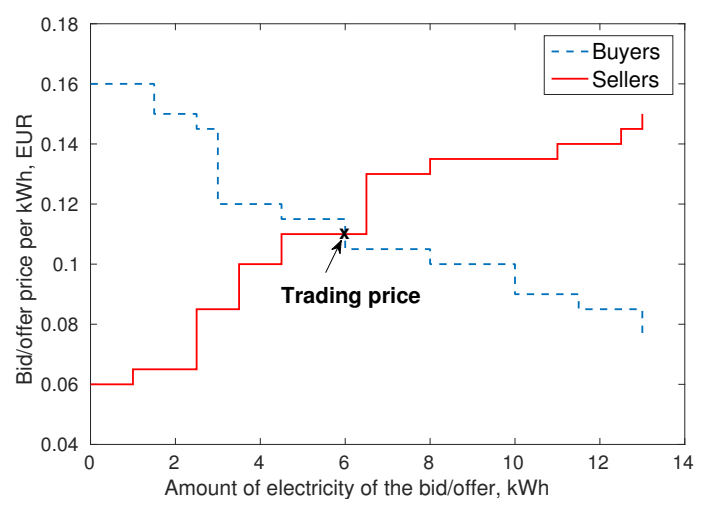

Fig. 3. Example of a double auction trading mechanism.

the trading price and amount of electricity traded on the local market, and (ii) which users will trade on the market, i.e., the sellers whose offer price is lower or equal to the determined trading price and the buyers whose biding price is higher or equal to the trading price.

3) Informing users/suppliers: The market informs the users about the amount and price of the electricity they traded for the trading period. It also informs the suppliers of the trading users about the electricity agreed to be traded, so the suppliers can adjust their bids/offers on the wholesale electricity market accordingly (to avoid imbalance fines).

4) Delivering electricity: During the electricity trading period sellers (buyers) should export (import) the amount of electricity they sold (bought) on the local market. If the amount of electricity the trading users import or export is different than the amount they traded, the users in short (long) automatically buy (sell) the shortage of (extra) electricity from (to) their contracted supplier.

5) Calculating rewards/costs: At the end of the trading period, each SM measures its user's imported and exported electricity for this period, and reports these values to the DSO, TSO and the corresponding supplier.

6) Settling payments: Once the suppliers receive the imported and exported electricity values from their customers' SMs, they use them in conjunction with the users' trades for the trading period and the trading price to adjust the customers' bills in order to reflect the effect of the users participating in the local electricity market.

\section{Example of a Local Electricity Trade}

Suppose there are two users, $\mathrm{U}_{1}$ and $\mathrm{U}_{2}$, contracted with a supplier, S. Both users buy electricity from $\mathrm{S}$ for $0.2 € / \mathrm{kWh}$ (incl. network fee $0.03 € / \mathrm{kWh}$ ) and automatically sell any excess electricity to $\mathrm{S}$ for $0.04 € / \mathrm{kWh}$ (excluding network fee).

Suppose during a trading period $\mathrm{U}_{1}$ exports $2 \mathrm{kWh}$ electricity to the grid whereas $\mathrm{U}_{2}$ imports $4 \mathrm{kWh}$ from the grid. In a current electricity market, $\mathrm{U}_{1}$ will be paid $0.08 €$ by $\mathrm{S}$ for the $2 \mathrm{~kW}$ exported, $\mathrm{U}_{2}$ will pay $\mathrm{S} 0.80 €$ for the $4 \mathrm{~kW}$ imported from the grid and the DSO/TSO will be paid $0.18 €$ by $\mathrm{S}$ in network fees for the imported and exported electricity by

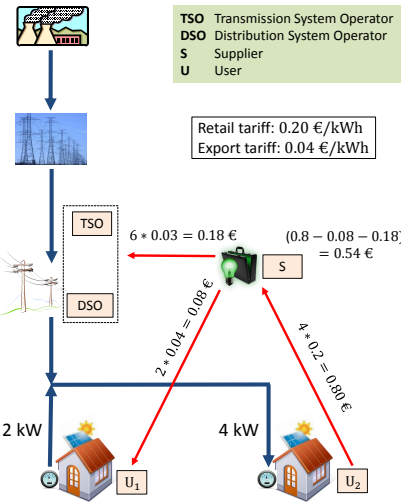

a) Without the use of a local market

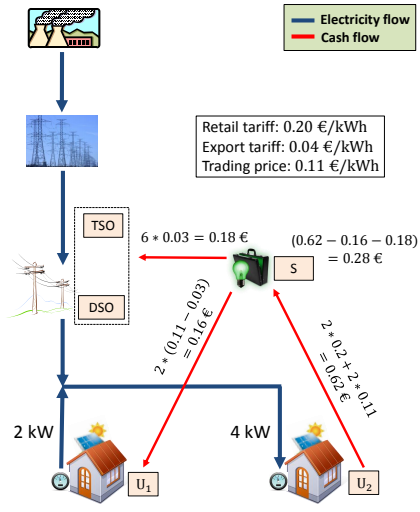

b) With the use of a local market
Fig. 4. Financial settlements among entities a) without using a local market, and b) with using a local market with trading price $0.11 € / \mathrm{kWh}$.

TABLE I

FinANCIAL SETTLEMENTS Without/With a Local Market (LM).

\begin{tabular}{|l|c|c|c|}
\hline & without LM & with LM & difference in \% \\
\hline $\mathrm{U}_{1}$ (seller) & $+0.08 €$ & $+0.16 €$ & $+100.00 \%$ \\
$\mathrm{U}_{2}$ (buyer) & $-0.80 €$ & $-0.62 €$ & $-22.50 \%$ \\
$\mathrm{DSO} / \mathrm{TSO}$ & $+0.18 €$ & $+0.18 €$ & $00.00 \%$ \\
$\mathrm{~S}$ (supplier) & $+0.54 €$ & $+0.28 €$ & $-51.85 \%$ \\
\hline
\end{tabular}

both users, leaving $\mathrm{S}$ with $0.54 €$ revenue (see Fig. $4 \mathrm{a}$ ). Now suppose that both users trade on a local market, i.e., $U_{1}$ and $\mathrm{U}_{2}$ trade $2 \mathrm{kWh}$ for a trading price $0.11 € / \mathrm{kWh}$, for example. In this case, $\mathrm{U}_{1}$ will be paid $0.16 €$ for the $2 \mathrm{~kW}$ exported by $\mathrm{U}_{2}$ via $\mathrm{S}, \mathrm{U}_{2}$ will pay $\mathrm{S} 0.62 €$ for the $4 \mathrm{~kW}$ imported (for 2 $\mathrm{kW}$ to each, $\mathrm{S}$ and $\mathrm{U}_{1}$ (via $\mathrm{S}$ )) and the DSO/TSO will be paid $0.18 €$ by $S$, leaving $S$ with $0.28 €$ revenue (see Fig. $4 b$ ).

From the example it is clear that users will benefit financially from our proposed local market. If they trade on the local market, users will be paid more for their exported electricity and pay less for their imported electricity. The DSO/TSO will not be affected as they will be paid the same regardless of the amount of electricity traded on the local market. A comparison of the financial settlements in our example with users (not) trading on the local market is given in Table I.

\section{E. Benefits of the Proposed Local Electricity Market}

Our proposed electricity market will have various benefits which can be grouped into two categories: financial and environmental benefits. Table II lists some of these benefits.

1) Financial Benefits: Our proposed local market would allow (i) users to sell their excess electricity for a price higher than the import tariff offered by their contracted suppliers, thus increasing their revenues from RESs, and (ii) users to buy electricity for a price cheaper than the retail price offered by their suppliers, thus reducing their bills. Moreover, trading electricity locally (iii) would reduce the transmission costs/losses contributing also towards lower electricity prices, and (iv) reduce the need for building new transmission lines.

2) Environmental Benefits: As our market model encourages local user-to-user electricity trades, less electricity will be 
TABLE II

Benefits of OUR Proposed LOCAL Electricity MARKET.

\begin{tabular}{|l|l|}
\hline Financial Benefits & Environmental Benefits \\
\hline More revenue for RES users & Less congestion at transmission lines \\
Reduces bills for users & Less use of conventional generators \\
Reduced transmission costs & Reduced use of transmission lines \\
Reduced electricity price & Reduced transportation loses \\
Fewer new transmission lines & Reduced operational costs \\
\hline
\end{tabular}

(i) generated at far-off generators, (ii) transported at transmission lines and (iii) lost at the transmission level. As a result less electricity will be generated by conventional generators leading to less greenhouse gas emissions. Instead, sources of clean energy, i.e., RESs, will be more popular and used widely.

\section{THREAT ANALYSiS}

Although a local electricity market could bring financial benefits to users and environmental benefits in general, it may also create an opportunity for some entities to misbehave in order to reduce costs or maximize profits [14]. This section analyses such cases, and lists potential security/privacy threats.

Impersonation. A malicious consumer may impersonate another user and offer a very low bid in his/her place in order to win a good offer to eventually reduce his/her own electricity bill, since the price of electricity traded at the local market is lower than the retail price. By the same token, it may also be the case that a user impersonates others and submit a high offer in their place to the market in order to win a bid. Therefore, it is important to have a proper user (or entity) authentication mechanism put in place.

Data manipulation. A malicious user may attempt to modify the content of other users' data (e.g., how much electricity they can offer at what price and so on) and provide inaccurate information in order to lower their credibility in the market. In addition, a misbehaving supplier may also attempt to modify users' offers/bids in attempt to manipulate the local market for its own benefit. Therefore, a secure digital signature scheme is needed to ensure the integrity and authenticity of messages.

Eavesdropping. An adversary may attempt to eavesdrop messages sent to the market. Such messages may include sensitive data such as user identity, contracted suppliers, meter readings, etc. The adversary may use such data to impersonate a user or to learn users' electricity capacity in order to gain a competitive advantage in the market. In addition, by observing who is selling how much electricity in the local market at a given time period, one may be able to learn, among other, whether someone is at home. This constitutes a privacy threat to the users, and may also incur additional risks, e.g., burglary. Hence, confidentiality of such information must be guaranteed using a secure encryption scheme. In addition, a secure access control and authorisation mechanism are needed too.

Privacy Breaches. Providing protection against unauthorised entities may not be sufficient to preserve users' privacy. Legitimate entities (e.g., the local market, DSO/TSO, suppliers) which have access to users' sensitive data may use such data for purposes that are not directly relevant to the local electricity trading. For example, entities that have access to users' offers/bids may use such data to infer information such as who, when and how much electricity is selling/buying. Such data is closely correlated to users' consumption data/patterns which have been shown to be a privacy concern for users [15]. Hence, privacy enhancing technologies should be used to limit the access of legitimate entities to users' sensitive data.

Disputes. Disputes are inevitable in almost all markets, and the proposed local market is no exception. Disputes may arise when a user claims to consume less than she actually consumed, or when she claims to sell more than she actually sold in the market. It may also arise when someone repudiates the agreed upon price for the electricity. Therefore, a robust dispute resolution is also a must in the proposed market.

Denial-of-Service (DoS). DoS attacks aim to make services inaccessible to legitimate users. In a local electricity market context, DoS attacks can be targeted at the market itself (making it inaccessible to all users) or individual users' SMs (preventing these users from trading on the market). Such DoS attacks could be performed by external adversaries aiming to disrupt the normal operation of the market, or misbehaving suppliers aiming to shut down the entire market to prevent users from trading among each others, or block specific users in order to buy their excess electricity at a cheap price instead of allowing them to trade on the market. Thus, measures should be in place to mitigate DoS attacks.

\section{Security and Privacy Requirements}

Based on the threat analysis, this section specifies a set of security and privacy requirements for local electricity markets.

Entity Authentication is important to ensure that entities can be assured of the identity of their communication partner. It is used to counter impersonation attacks. A liveness guarantee, i.e., the fact that the entity is active during authentication, is also an essential part of entity authentication. Entity authentication can be achieved by using a challenge-response protocol, so replaying eavesdropped responses can be detected.

Message Authenticity guarantees an entity that the message it received has not been tampered with while in transit. It is used to detect message modifications. It can be achieved by means of a digital signature or a message authentication code. The advantage of digital signatures is that they are based on asymmetric keys, thus they also provide non-repudiation. Moreover, the integrity of the software running on SMs should be guarantied by using protected module architectures as suggested in [16] and implemented in [17].

Authorisation is a process of determining if an entity has permissions to use/access resources, known as access control too. It can be coupled with entity authentication so the authorising party is aware of the identity of the entity requesting access. It is used to counter elevation of privilege attacks.

Confidentiality ensures that only the intended receiver(s) of a message can read the message. It is used to counter eavesdropping attacks. Confidentiality is achieved by encryption. Both symmetric and asymmetric encryption are possible, with 
symmetric encryption having the advantage of being less computationally heavy. Message authentication and confidentiality can be combined when using authenticated encryption.

User Privacy-preservation ensures that user privacy is protected as much as possible. To achieve this the 'principle of least privilege', i.e., only allow an entity to have access to data just sufficient for it to carry out its duties, should be applied. For example, the local market does not need to know the identities of users trading on the market, as long as it is assured that they are legitimate users. This can be achieved by combining anonymity, non-linkability and anonymous signature. Anonymity can be achieved using pseudonyms, which also offer non-linkability if a unique one-time pseudonym is used by each user for each trading period (as in [18]). Anonymous signature could be achieved by using group signature or ring signature schemes. The market could also incorporate privacy-friendly services empowered by a blockchain protocol, such as Enigma [19], or secure multiparty computation as in [20]. Moreover, a supplier does not need to know exactly which of its customers traded how much electricity for which trading period on the local market. It should be sufficient for the supplier just to know (i) how much electricity in total its customers traded at each period, and (ii) how much per month it should reward/charge each of its customers for the electricity traded on the market. To achieve this, a combination of selective data aggregation using homomorphic encryption (as in [21]), commitment scheme and zero-knowledge proofs (as in [22], [23]) could be used.

Non-repudiation is achieved when an entity cannot deny having sent a message when it did indeed send that message. This can only be achieved when messages are authenticated using a cryptographic key that only one entity has access to, i.e., using asymmetric cryptography.

Availability is used to ensure that a system or a system resource is accessible upon demand by authorised entities. It is used to counter DoS attacks. Availability can be achieved by using a combination of load and resource balancing, attack detection, message classification and filtering techniques.

\section{CONCLUSION}

In this paper we presented a local electricity trading market in which RES owners can sell their excess electricity either to other users in their neighbourhood or to suppliers, based on a system of bidding. This leads to a significant financial gain for the RES owners and consumers, as well as ecological benefits. We then performed a threat analysis of such a market and specified a set of security and privacy requirement which such markets should satisfy. This set of requirements can be used as a guide for secure and privacy-preserving protocol design. As a future work we will be working towards designing such a protocol to support local electricity markets.

\section{ACKNOWLEDGMENT}

This work was supported in part by the Research Council KU Leuven: C16/15/058, by the European Commission through FP7 Harmonized framework allowing a sustainable and robust identity for European Citizens (EKSISTENZ)," with grant number: 607049 and by KIC InnoEnergy SE through KIC Innovation Project SAGA.

\section{REFERENCES}

[1] H. Farhangi, "The Path of the Smart Grid," in IEEE Power Energy Magazine, vol. 8, no. 1, pp. 18-28, Jan./Feb. 2010.

[2] Vergoeding overtollige elektriciteit? [Online]. Available: http://www.vreg. be/nl/vergoeding-overtollige-elektriciteit, accessed Apr. 1, 2016.

[3] Feed-in tariffs: get money for generating your own electricity. [Online]. Available: https://www.gov.uk/feed-in-tariffs, accessed Mar. 16, 2016.

[4] W. Saad, Zhu Han, H. V. Poor and T. Başar, "Game-theoretic Methods for the Smart Grid: An Overview of Microgrid Systems, Demandside Management, and Smart Grid Communications," in IEEE Signal Processing Magazine, vol. 29, no. 5, pp. 86-105, Sept. 2012.

[5] Perukrishnen Vytelingum, Sarvapali D. Ramchurn, Thomas D. Voice, Alex Rogers, and Nicholas R. Jennings, "Trading Agents for the Smart Electricity Grid," in 9th Int. Conf. on Autonomous Agents and Multiagent Systems (AAMAS), pp. 897-904, May 2010.

[6] W. Lee, L. Xiang, R. Schober and V. W. S. Wong, "Direct Electricity Trading in Smart Grid: A Coalitional Game Analysis," in IEEE Journal on Selected Areas in Communications, vol. 32, no. 7, pp. 1398-1411, 2014

[7] J. Lee, J. Guo, J. K. Choi and M. Zukerman, "Distributed Energy Trading in Microgrids: A Game-Theoretic Model and Its Equilibrium Analysis," in IEEE Transactions on Industrial Electronics, vol. 62, no. 6, pp. 35243533, June 2015

[8] "The electricity trading arrangements: A beginners guide," Elexon, Technical Report, November 2015. [Online]. Available: http://bit.ly/1MBHc5s, accessed Mar. 16, 2016.

[9] Annual domestic energy bills [Online]. Available: http://tinyurl.com/ o8zybas, accessed Mar. 16, 2016.

[10] N. Yaagoubi and H. T. Mouftah, "Energy Trading in the Smart Grid: A Game Theoretic Approach," in IEEE Int. Conf. on Smart Energy Grid Engineering (SEGE), pp. 1-6, 2015.

[11] W. Tushar, C. Yuen, D. B. Smith and H. V. Poor, "Price Discrimination for Energy Trading in Smart Grid: A Game Theoretic Approach," in IEEE Transactions on Smart Grid, vol. PP, no. 99, pp. 1-12.

[12] M. Ampatzis, P. H. Nguyen and W. Kling, "Local Electricity Market Design for the Coordination of Distributed Energy Resources at District Level," in IEEE PES Conference on Innovative Smart Grid Technologies Europe (ISGT-Europe), pp. 1-6, 2014.

[13] I. S. Bayram, M. Z. Shakir, M. Abdallah and K. Qaraqe, "A Survey on Energy Trading in Smart Grid," in IEEE Global Conference on Signal and Information Processing (GlobalSIP), pp. 258-262, 2014.

[14] G. Kalogridis, M. Sooriyabandara, Z. Fan and M. A. Mustafa, "Toward Unified Security and Privacy Protection for Smart Meter Networks,' in IEEE Systems Journal, vol. 8, no. 2, pp. 641-654, June 2014.

[15] E. L. Quinn, "Privacy and the new energy infrastructure," in Social Science Research Network (SSRN), pp. 1-41, Feb. 2009.

[16] S. Cleemput, M. A. Mustafa and B. Preneel, "High Assurance Smart Metering," in IEEE Int. Symposium on High Assurance Systems Engineering (HASE), 2016, pp. 294-297.

[17] J. T. Mühlberg, S. Cleemput, M. A. Mustafa, J. Van Bulck, B. Preneel and F. Piessens, "An Implementation of a High Assurance Smart Meter using Protected Module Architectures," in Int. Conf. on Information Security Theory and Practice (WISTP), Springer, 2016.

[18] M. A. Mustafa, N. Zhang, G. Kalogridis and Z. Fan, "Roaming electric vehicle charging and billing: An anonymous multi-user protocol," in IEEE Int. Conf. on Smart Grid Communications, 2014, pp. 939-945.

[19] Guy Zyskind, Oz Nathan and Alex 'Sandy' Pentland, "Enigma: Decentralized Computation Platform with Guaranteed Privacy," whitepaper. [Online]. Available: http://bit.ly/1TkEJ0I, accessed Mar. 29, 2016.

[20] A. Aly and M. Van Vyve, "Practically efficient secure single-commodity multi-market auctions," in Financial Cryptography (FC), LNCS, 2016.

[21] M. A. Mustafa, N. Zhang, G. Kalogridis and Z. Fan, "DEP2SA: A Decentralized Efficient Privacy-Preserving and Selective Aggregation Scheme in Advanced Metering Infrastructure," in IEEE Access, vol. 3, pp. 2828-2846, 2015.

[22] A. Rial and G. Danezis, "Privacy-preserving smart metering," in $A C M$ Workshop on Privacy in the Electronic Society (WPES), pp. 4960, 2011.

[23] G. Danezis, M. Kohlweiss, and A. Rial, "Differentially private billing with rebates," in Information Hiding, LNCS, vol. 6958, pp. 148-162, 2011. 